\title{
ERRATA
}

\section{THEORY OF LATTICE SPECIFIC HEAT OF AN ISOTOPICALLY DISORDERED ANHARMONIC CRYSTAL}

\author{
B. D. Indu
}

[INT. J. MOD. PHYS. B, Vol. 4, Nos. $7 \& 8$ (1990) 1379-1393]

Page 1382: $\eta_{i}$ in Eq. (10c) should be read as $\eta_{i-1}$.

Page 1388, last paragraph: $(1-c)^{2} I(8)$ and $(1-c) I(6)$ should be read as $c(1-c) I(8)$ and $c I(6)$, respectively.

Appendix A, pages 1390-91: Equation (A.1) should be read as

$$
\begin{aligned}
\Gamma_{k}^{D}(\omega) & =\left(V / 8 \pi \nu^{3}\right)\left(M_{0} / N \mu\right)^{2}\left(\omega_{k_{1}} / \omega_{k}\right) c\left[(1-c) \omega_{k_{1}}^{4}-2 \mu\left(\omega_{k_{1}} / M_{0}\right)^{2}\right. \\
& \left.\times \sum_{11^{\prime}} \Delta \phi_{11^{\prime}}\left(1-\Delta \phi_{11^{\prime}} / 3 M_{0}^{2} \omega_{k}^{2} c\right)\right]
\end{aligned}
$$

Consequently, in Eqs. (A.6), (A.7), (A.8), (A.10), (A.11) and (A.13), $(1-c)^{2}$ should be read as $c(1-c)$ and $(1-c)$ as $c$.

Page 1392: $\left(2 n_{k}+1\right)$ should be read as $2 n\left(\omega_{k}\right)+1$ and Vol. 13 in Ref. 21 should be read as Vol. 139. 